\title{
EFICÁCIA E SELETIVIDADE DO HERBICIDA PROPISOCHLOR APLICADO EM PRÉ E PÓS-EMERGÊNCIA, ISOLADO E EM MISTURAS, SOBRE DIFERENTES HÍBRIDOS DE MILHO
}

\author{
Marcus Barifouse Matallo ${ }^{1}$, Eduardo Antonio Drolhe da Costa ${ }^{1}$, \\ Flávio Martins Garcia Blanco², Ednei de Conti Macedo² e Albino Rozanski³
}

${ }^{1}$ Eng. Agrônomo, Dr., Pesquisador Científico. Instituto Biológico, Laboratório das Ciências das Plantas Daninhas. Caixa Postal 70. Campinas, SP, 13001-970. matallo@biologico.br

${ }^{2}$ Eng'. Agrônomo, M.Sc., Pesquisador Científico.

${ }^{3}$ Eng. Agrônomo, Pesquisador Científico.

\section{RESUMO}

Foram realizados dois experimentos de campo para verificar a eficácia do herbicida propisochlor aplicado em pré e pósemergência no controle de plantas daninhas e a seletividade sobre quatro híbridos de milho. Os experimentos foram conduzidos no município de Engenheiro Coelho, SP, com os tratamentos em pré-emergência aplicados em 25/11/97 e os de pós-emergência em 19/12/97. Utilizou-se o delineamento experimental de parcelas subdivididas em blocos ao acaso, com arranjo sistemático das parcelas principais (cultivares), com quatro repetições e os seguintes tratamentos: propisochlor (nas doses de 1008,1080 e 1152 $\mathrm{g} / \mathrm{ha}$, propisochlor/atrazine (a 1008/1400,1080/1400 e 1152/1400 g/ha), propisochlor + terbutylazine (a 1008+1800, 1080+1800 e $1152+1800 \mathrm{~g} / \mathrm{ha})$, acetochlor (2688 g/ha), metolachlor (1250 g/ha), atrazine (3500 g/ha), terbutylazine (3000 g/ha), uma testemunha sem capina e outra capinada para as avaliações de controle e seletividade, respectivamente. Os resultados demonstraram que o herbicida propisochlor isolado, ou em mistura com atrazine ou terbutylazine, controlou eficientemente as espécies Brachiaria plantaginea, Digitaria horizontalis, Eleusine indica, Amaranthus viridis e Bidens pilosa, até 45 dias após sua aplicação em pré-emergência das ervas. A espécie Brachiaria decumbens apresentou níveis de controle eficientes somente quando o propisochlor foi aplicado em mistura com atrazine ou terbutylazine. Aplicado em pós-emergência, os níveis de controle de propisochlor isolado ou em mistura com os demais herbicidas foram insuficientes. Não foram observados sintomas visuais de injúrias nem redução significativa no rendimento dos híbridos C-444, C-505, C-901 e C-909 por ação tóxica do propisochlor, tanto isoladamente como em mistura formulada com atrazine ou em tanque com terbutylazine.

Palavras-chave: mistura de herbicidas, plantas daninhas, Zea mays.

\section{ABSTRACT \\ Effect of propisochlor with and without other herbicides in pre and post-emergence in corn}

Two field experiments were carried out in Engenheiro Coelho, SP, to evaluate the selectivity and efficiency of the herbicide propisochlor, alone and in combination with other herbicides, applied in pre and post-emergence in corn. A randomized block design was used with four replications including the following treatments: propisochlor (at 1008, 1080 and 1152 g/ha), propisochlor formulated with atrazine (at 1008/1400,1080/1400 e 1152/1400 g/ha), and with terbutylazine in tank mix (at 1008+1800, $1080+1800 \mathrm{e} 1152+1800 \mathrm{~g} / \mathrm{ha}$ ), acetochlor at $2688 \mathrm{~g} / \mathrm{ha}$, metolachlor at $1250 \mathrm{~g} / \mathrm{ha}$, atrazine at $3500 \mathrm{~g} / \mathrm{ha}$; terbutylazine at $3000 \mathrm{~g} / \mathrm{ha}$ and controls with and without application products for selectivity and control respectively. The results showed that propisochlor alone and in mixture with atrazine or terbutylazine at any dose controlled efficiently Brachiaria plantaginea, Digitaria horizontalis, Eleusine indica, Amaranthus viridis and Bidens pilosa, up to 45 days with pre application. Propisochlor only in combination with either atrazine or terbutilazine did not have satisfactory post-emergence activity. None of the chemical treatments affected corn growth.

Key words: herbicide mixtures, propisochlor, weed, Zea mays. 


\section{INTRODUÇÃO}

A cultura do milho no Brasil vêm apresentando melhoria no seu nível tecnológico, com crescentes ganhos de produtividade nas principais regiões produtoras. Os aumentos de produtividade da cultura resulta dos esforços da pesquisa agronômica em diferentes áreas tais como genética e melhoramento, adubação e calagem, práticas culturais, controle de pragas e doenças e pesquisas biológicas básicas ao longo dos últimos 60 anos.

A cultura do milho não pode sofrer interferência de plantas daninhas até o pendoamento (Silva \& Silva, 1987), pois provocam perdas que variam de 12 a 85\%, devido à competição (Cruz \& Ramalho, 1983). No Estado de São Paulo, verificaram-se reduções de $70 \%$ nas lavouras de milho não capinadas (Blanco et al., 1976).

O uso contínuo de herbicidas no controle das plantas daninhas dicotiledôneas em milho, girassol, batata, soja e beterraba açucareira, juntamente com a rotação de culturas, pode ter influência no crescimento em grande escala de gramíneas, como as dos gêneros Echinochloa, Setaria e Digitaria. Nesse contexto, o novo herbicida propisochlor pode solucionar esse problema, controlando as mono e dicotiledôneas.

O propisochlor é um herbicida seletivo, do grupo das acetanilidas, aplicado em pré-emergência ou em pré-plantio incorporado. É utilizado para o controle de gramíneas anuais e de algumas plantas daninhas do grupo das dicotiledôneas que infestam as culturas de milho, girassol, soja, batata e ervilha. O desenvolvimento de formulações com protetores em alguns herbicidas desse grupo, como o alachlor e o metolachlor, ampliam as possibilidades de uso agrícola desses compostos como, por exemplo, em sorgo e milho (Foster, 1997).

Absorvido preferencialmente pelas raízes nas dicotiledôneas e pelo caulículo nas monocotiledôneas, tem sua translocação bastante limitada tanto através do xilema como do floema. A molécula do propisochlor apresenta baixa solubilidade em água (154 mg/l), sendo pouco lixiviável através do perfil do solo. De uma forma geral, as acetanilidas são adsorvidas pelos colóides orgânicos e inorgânicos do solo, sendo preferencialmente degradadas pelos microrganismos, apresentando uma persistência no solo que varia entre 8 e 12 semanas. Devido à sua baixa pressão de vapor, as perdas por volatilização desses herbicidas podem ser consideradas desprezíveis (Foster, 1997).

No Brasil, a molécula do propisochlor não está registrada para uso agrícola e quase nada se conhece a respeito de sua eficiência agronômica, assim como de seu comportamento no ambiente, e em particular no solo.

O objetivo deste trabalho foi o de avaliar a eficácia do propisochlor no controle de plantas daninhas mono e dicotiledôneas e sua seletividade a diferentes híbridos de milho.

\section{MATERIAL E MÉTODOS}

Dois experimentos foram conduzidos durante o período de 20 de novembro de 1997 a 2 de março de 1998, no município de Engenheiro Coelho, SP, com o herbicida propisochlor para verificar sua eficácia no controle de plantas daninhas e sua seletividade a quatro novos híbridos de milho (C-444, C505, C-901 e C-909) quando aplicado em pré e pós-emergência.

A área experimental está situada a $22^{\circ} 30^{\prime}$ de latitude sul e $47^{\circ} 10^{\prime}$ de longitude oeste e a $645 \mathrm{~m}$ de altitude, apresentando um clima subtropical e solo com textura argilosa, pertencente à classe Latossolo com 49,0\% de argila, 14,7\% de areia grossa, 30,5\% de areia fina e 5,7\% de limo. A análise química mostrou pH $\left(\mathrm{CaCl}_{2}\right)$ de 5,2, $18 \%$ de M.O., $30 \mathrm{mg} / \mathrm{dm}^{3}$ de P (resina), 2,5 mmol $/ \mathrm{dm}^{3}$ de K, $35 \mathrm{mmol}_{\mathrm{c}} / \mathrm{dm}^{3} \mathrm{de} \mathrm{Ca}, 12$ $\mathrm{mmol} / \mathrm{dm}^{3} \mathrm{de} \mathrm{Mg}, 31 \mathrm{mmol} / \mathrm{dm}^{3} \mathrm{de} \mathrm{Al}+\mathrm{H}, 80,5 \mathrm{de}$ CTC e V de $61,4 \%$. O preparo do solo constou de uma aração e duas gradagens cruzadas, sendo a última realizada um dia antes do plantio.

Os experimentos em pré e pós-emergência foram conduzidos em parcelas experimentais formadas de modo a se verificar independentemente a seletividade e o controle das plantas daninhas. Para a verificação da seletividade, as parcelas foram formadas por duas linhas de cada híbrido espaçadas de 0,80 m, com 4,0 m de comprimento, semeados numa densidade de 6 a 8 sementes por metro linear, perfazendo uma área útil de $22,4 \mathrm{~m}^{2}$ por unidade experimental. As parcelas para a verificação da eficácia de controle foram formadas por duas linhas de cada espécie semeadas manualmente e espaçadas de $0,40 \mathrm{~m}$, com 4,0 m de comprimento, totalizando uma área útil de $17,6 \mathrm{~m}^{2}$. A adubação foi realizada com $400 \mathrm{~kg} / \mathrm{ha}$ da fórmula 04-14-08 na linha de semeadura e aproximadamente 30 dias após a germinação aplicou-se $40 \mathrm{~kg} /$ ha de uréia em cobertura.

$\mathrm{O}$ experimento foi instalado num delineamento de parcelas subdivididas em blocos ao acaso, com arranjo sistemático das parcelas principais (cultivares) e repetidos quatro vezes.

Foi realizada uma única aplicação de cada tratamento em 25/11/97 no experimento de pré-emergência e em 19/12/97 no de pós-emergência (Tabela 1). A Tabela 2 apresenta os estádios e a densidade das plantas daninhas por ocasião da aplicação dos tratamentos em pós-emergência.

O volume de calda utilizado variou de 200 a $250 \mathrm{~L} / \mathrm{ha}$, empregando-se um pulverizador costal manual com pressão constante fornecida por $\mathrm{CO}_{2}$ comprimido à pressão de $31 \mathrm{kgf} /$ $\mathrm{cm}^{2}$, equipado com quatro pontas de pulverização do tipo jato plano Teejet DG $110-01$, espaçados de $0,5 \mathrm{~m}$, proporcionando uma faixa de aplicação de $2,0 \mathrm{~m}$. As condições ambientais durante as aplicações nos ensaios de pré e pós-emergência foram, respectivamente, de 26 e $28^{\circ} \mathrm{C}$ (temperatura do ar), $78 \mathrm{e}$ $81 \%$ (umidade relativa do ar), ventos de 4 e $5 \mathrm{~km} / \mathrm{h}$ e $19,5 \%$ de umidade do solo no ensaio de pré-emergência. 
Eficácia e seletividade do herbicida propisochlor aplicado em pré e pós-emergência, isolado e em misturas, sobre diferentes híbridos de milho

Tabela 1. Tratamentos e doses utilizadas nos experimentos em pré e pós-emergência. Engenheiro Coelho, SP. $1997 / 1998$.

\begin{tabular}{lcc}
\hline \multicolumn{1}{c}{ Tratamentos } & Dose \\
\cline { 2 - 3 } \multicolumn{1}{c}{ Nome Técnico (produto comercial) } & i.a. (g/ha) & p.c. (l/ha) \\
\hline Propisochlor (SIP 015) & 1008 & 1,4 \\
Propisochlor (SIP 015) & 1080 & 1,5 \\
Propisochlor (SIP 015) & 1152 & 1,6 \\
Propisochlor/atrazine (SIP 015 + Gesaprim 500) & $1008+1400$ & $1,4+2,8$ \\
Propisochlor/atrazine (SIP 015 + Gesaprim 500) & $1080+1400$ & $1,5+2,8$ \\
Propisochlor/atrazine (SIP 015 + Gesaprim 500) & $1152+1400$ & $1,6+2,8$ \\
Propisochlor + terbutylazine (SIP 015 + SIP 05) & $1008+1800$ & $1,4+3,6$ \\
Propisochlor + terbutylazine (SIP 015 + SIP 05) & $1080+1800$ & $1,5+3,6$ \\
Propisochlor + terbutylazine (SIP 015 + SIP 05) & $1152+1800$ & $1,6+3,6$ \\
Acetochlor (Fist) & 2688 & 3,2 \\
Metolachor (Kadet) & 1250 & 2,5 \\
Atrazine (Gesaprim 500) & 3500 & 7,0 \\
Terbutylazine (SIP 05) & 3000 & 6,0 \\
Testemunha sem capina ${ }^{1}$ & - & - \\
Testemunha capinada ${ }^{2}$ & - & -
\end{tabular}

\begin{tabular}{ll}
\hline i.a.:ingrediente ativo & ${ }^{1}$ No ensaio para verificação de controle \\
p.c. produto comercial & ${ }^{2}$ No ensaio para verificação da seletividade
\end{tabular}

Tabela 2: Estádio e densidade das plantas daninhas por ocasião da aplicação dos tratamentos em pós-emergência. Engenheiro Coelho, SP. 1997/1998.

\begin{tabular}{lcl}
\hline Espécie & $\begin{array}{c}\text { Densidade } \\
\text { (plantas/metro linear) }\end{array}$ & $\begin{array}{c}\text { Estádio de } \\
\text { Desenvolvimento }\end{array}$ \\
\hline Brachiaria decumbens & 32 & $2-4$ folhas c/ $9 \mathrm{~cm}$ altura \\
Brachiaria plantaginea & 20 & $2-4$ folhas c/ no máximo $10 \mathrm{~cm}$ \\
Eleusine indica & 24 & $2-3$ folhas com no máximo $3 \mathrm{~cm}$ \\
Digitaria horizontalis & 23 & $2-4$ folhas com no máximo $9 \mathrm{~cm}$ \\
Bidens pilosa & 183 & $2-3$ folhas c/ no máximo $5 \mathrm{~cm}$ \\
Amaranthus hibridus & 190 & $2-3$ folhas c/ no máximo $6 \mathrm{~cm}$ \\
\hline
\end{tabular}

Durante todo o ciclo da cultura foram realizados os tratamentos fitossanitários necessários antes das pragas alcançarem o Nível de Dano Econômico (NDE).

A avaliação dos tratamentos sobre as plantas daninhas foi realizado ao $45 \mathrm{DAT}$, no final do período de competição, de acordo com Blanco et al. (1976), segundo a escala de conceitos que varia de zero (nenhum controle) a $100 \%$ (controle total). Considerou-se conmo eficiente o controle de $80 \%$ (índice mínimo de controle-IMC) da população de cada espécie de planta daninha, não sendo portanto realizada a análise estatística desses dados. Por sua vez, a seletividade dos herbicidas à cultura foi avaliada pela análise estatística da produção de grãos com os dados submetidos ao teste $\mathrm{F}$ ao nível de $5 \%$ de probabilidade. Além disso, a ação fitotóxica dos herbicidas sobre a cultura foi avaliada por observações
Tabela 3. Escala de toxicidade de plantas provocada por herbicidas (ALAM, 1974 modificada).

\begin{tabular}{cl}
\hline Notas defitotoxicidade & \multicolumn{1}{c}{ Descrição das injúrias } \\
\hline 1 & Plantas normais, inguais à testemunha \\
2 & Plantas com isntomas leves de injúrias \\
3 & Plantas com sintomas moderados de injúrias \\
4 & Plantas com sintomas severos de injúrias \\
5 & Plantas inteiramente mortas \\
\hline
\end{tabular}

visuais dos sintomas de injúrias nas plantas das parcelas tratadas em comparação com as plantas desenvolvidas nas parcelas-testemunha capinadas e classificadas de acordo com a escala de notas de fitotoxicidade (ALAM, 1974, modificada) apresentada na Tabela 3 . 


\section{RESULTADOS E DISCUSSÃO}

As Figuras 1, 2 e 3 apresentam o desempenho dos tratamentos sobre as espécies infestantes, em percentagem de controle, nas aplicações de pré-emergência e as Figuras de 4, 5 e 6, nas aplicações em pós-emergência.

O desempenho do propisochlor quando aplicado em pré-emergência, tanto isoladamente como em mistura com atrazine ou terbutylazine, foi superior para algumas espécies, quando comparado com a aplicação deste produto em pósemergência. No caso de sua aplicação em pré-emergência, os níveis de controle de $B$. plantaginea são superiores ao IMC $(80 \%)$ nas três doses testadas isoladamente, atingindo valores de $95 \%$ na sua maior dose, o que pode ser considerado como um controle excelente; entretanto, a adição de atrazine ou terbutylazine provocou um incremento nos níveis de controle dessa espécie, elevando-o consideravelmente, chegando a alcançar níveis de controle total (100\%). Comparan-

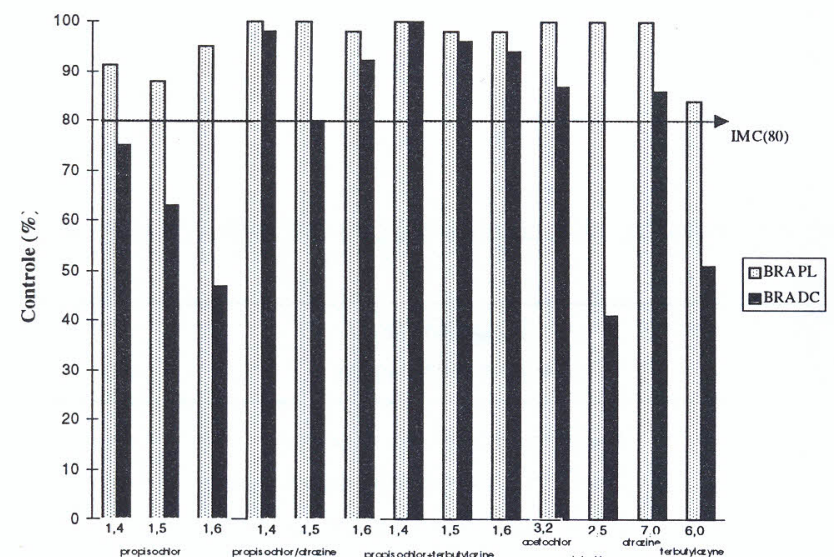

Figura 1. Controle de $B$. plantaginea e $b$. decumbens aos 45 DAT em pré-emergência.

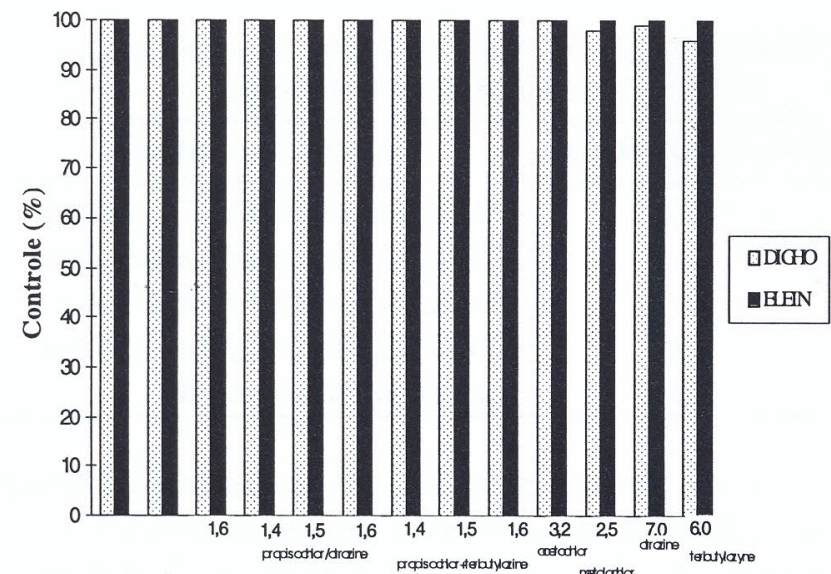

Figura 2. Controle de D. horizontalis e $E$. indica aos 45 DAT em pré-emergência.

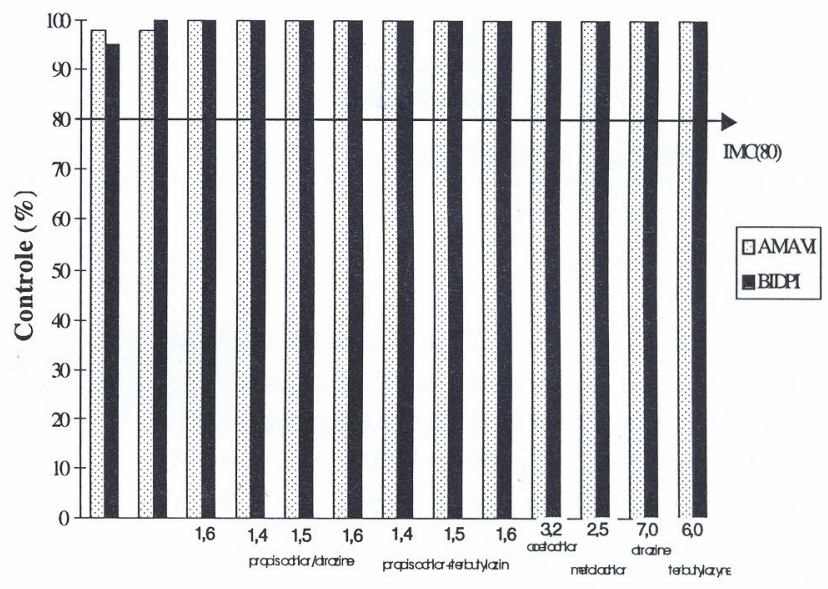

Figura 3. Controle de $A$. viridis e $B$. pilosa aos 45 DAT em pré-emergência.

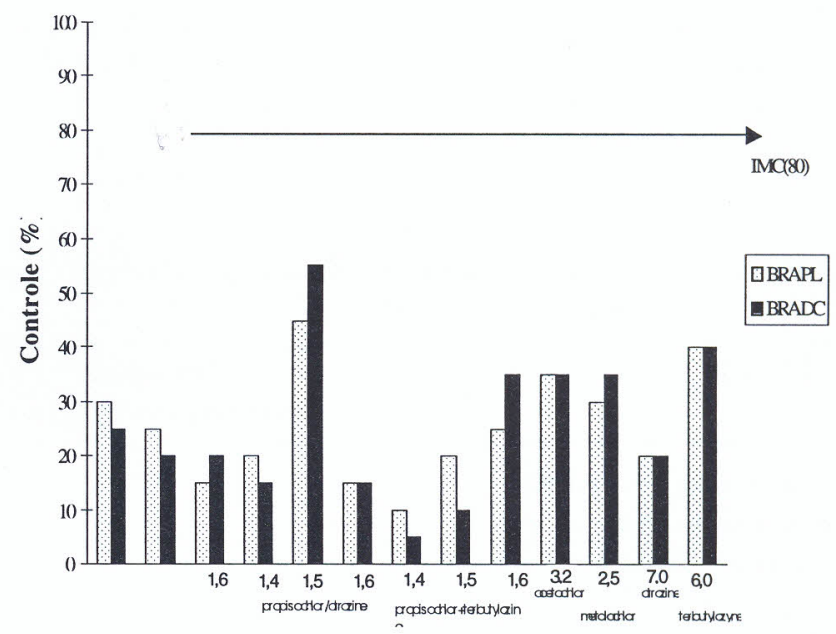

Figura 4. Controle de B. plantaginea e $b$. decumbens aos 45 DAT em pós-emergência.

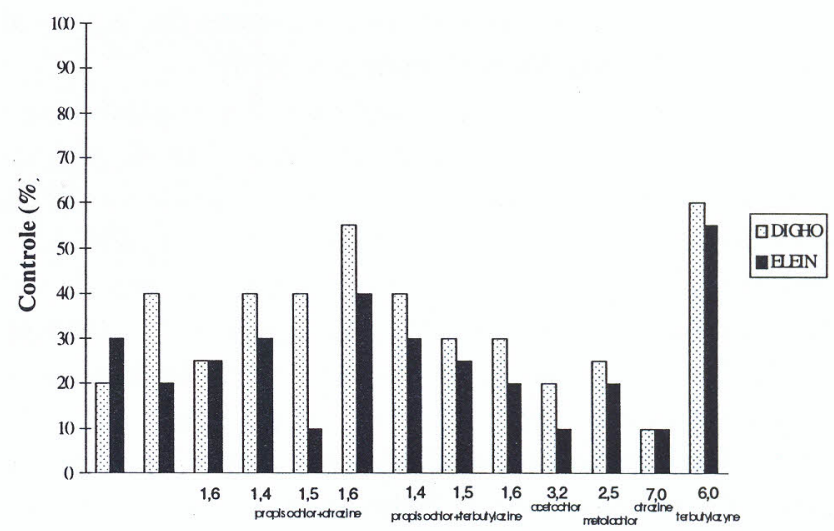

Figura 5. Controle de $D$. horizontalis e $E$. indica aos 45 DAT em pós-emergência. 
do-se com os níveis alcançados pelos herbicidas padrões utilizados no ensaio (acetochlor-100\% e metolachlor-100\%), o propisochlor isolado apresentou um desempenho inferior, tendo contudo, alcançado o mesmo nível quando em mistura em tanque com atrazine ou terbutylazine. Por outro lado, dos componentes dessas misturas, a atrazine, quando aplicada isoladamente, apresentou nível de controle similar àquele verificado quando em mistura com o propisochlor sendo, inclusive, superior a este nas doses testadas isoladamente. Já o terbutylazine sozinho apresentou o pior desempenho, muito embora o nível de controle obtido para essa espécie tenha sido, também, superior ao IMC (80\%).

Comportamento semelhante pôde ser observado com relação ao controle de $B$. decumbens, onde a adição de atrazine ou terbutylazine ao propisochlor provocou um incremento nos níveis de controle quando comparados àqueles verificados para esse herbicida isoladamente, os quais por sua vez, são inferiores ao IMC (80\%). O mesmo resultado foi observado por Carvalho et al.(2000), quando estudou o controle de $B$. decumbens com o metolachlor isolado ou em mistura pronta com atrazine, na dose de $2145 \mathrm{~g} / \mathrm{ha}$. A comparação com os padrões mostra um comportamento superior ao das misturas, assim como aos de seus componentes isolados.

Para as demais espécies ( $D$. horizontalis, E. indica, A. viridis e $B$. pilosa), o desempenho do propisochlor foi considerado excelente, independentemente se aplicado isoladamente ou em mistura com atrazine ou terbutylazine. Em qualquer situação ou em qualquer das doses testadas, os níveis de controle obtidos foram sempre superiores ao IMC (80\%) e quase sempre iguais a $100 \%$. A mesma eficiência sobre essas espécies foi observado por Queiroz et al. (1997) quando estudou na dose de $1800+1200 \mathrm{~g} / \mathrm{ha}$, o efeito do herbicida metolachlor em mistura com atrazine. Este mesmo modelo de comportamento foi observado para os herbicidas utilizados como padrões e, também, para os componentes das misturas quando aplicados isoladamente (atrazine e terbutylazine).

Nenhuma das espécies mostrou-se suscetível ao propisochlor quando este foi aplicado em pós-emergência, tanto isoladamente como em mistura com atrazine ou em tanque com terbutylazine, exceto no controle de $B$. pilos $a$. Almeida et al. (1988), também conseguiram controle satisfatório somente das ervas latifoliadas, quando utilizaram a mistura formulada de metolachlor/atrazine nas doses 1800/1200 g/ha, e de $B$. plantaginea na dose de $2400 / 1600 \mathrm{~g} / \mathrm{ha}$, aplicados em pós-emergência inicial das plantas daninhas e da cultura de milho.

As Tabelas 4 e 5 mostram a produção dos híbridos de milho tratados com os diferentes herbicidas, aplicados em pré e pós-emergência. A análise da variância dos dados de produção dos híbridos mostra que não foi detectado qualquer efeito dos tratamentos secundários (herbicidas) e testemunhas sobre a produção de grãos em qualquer dos cultivares (híbridos) estudados tanto em pré como em pós-emergência, caracterizando dessa forma a seletividade do propisochlor (isolado ou em mistura) e, também, dos demais produtos testados. Além disso, embora os dados não mostrem, em nenhum momento durante a condução dos ensaios foram observados sintomas de injúrias devido a ação tóxica dos produtos sobre as plantas de milho, com estas obtendo nota 1 durante todo seu ciclo.

Tabela 4. Produção dos híbrios de milho tratados com diferentes herbicidas aplicados em pré-emergência. Engenheiro Coelho, SP. $1997 / 1998$

\begin{tabular}{|c|c|c|c|c|c|}
\hline \multirow{3}{*}{ Tratamento } & \multirow{3}{*}{$\begin{array}{l}\text { Dose } \\
(\mathrm{g} / \mathrm{ha})\end{array}$} & \multicolumn{4}{|c|}{ Produção (kg/ha) } \\
\hline & & \multicolumn{4}{|c|}{ Híbridos } \\
\hline & & C-444 & C-505 & C-901 & C-909 \\
\hline Propisochlor & 1008 & 4687,5 & 5625,0 & 4687,5 & 5312,5 \\
\hline Propisochlor & 1080 & 4687,5 & 5468,6 & 5000,0 & 5625,0 \\
\hline Propisochlor & 1152 & 4843,7 & 6093,8 & 5625,0 & 5781,3 \\
\hline Propisochlor/atrazine & $1008+1400$ & 4531,3 & 5937,5 & 5312,5 & 5781,3 \\
\hline Propisochlor/atrazine & $1080+1400$ & 5000,0 & 6093,8 & 5625,0 & 5468,8 \\
\hline Propisochlor/atrazine & $1152+1400$ & 3906,3 & 5468,8 & 4375,0 & 5937,5 \\
\hline Propisochlor + terbutylazine & $1008+1800$ & 4531,3 & 6250,0 & 5000,0 & 5468,8 \\
\hline Propisochlor + terbutylazine & $1080+1800$ & $43,75,0$ & 5781,3 & 5468,8 & 5625,0 \\
\hline Propisochlor + terbutylazine & $1152+1800$ & 4843,7 & 5156,3 & 5468,8 & 5312,5 \\
\hline Acetochlor & 2.688 & 4531,3 & 5781,3 & 5000,0 & 5312,5 \\
\hline Metolachlor & 1250 & 5000,0 & 5937,5 & 4687,5 & 5468,8 \\
\hline Atrazine & 3500 & 4687,5 & 5625,0 & 5156,3 & 5781,3 \\
\hline Terbutylazine & 3000 & 4843,7 & 6406,3 & 5468,8 & 5781,3 \\
\hline Testemunha capinada & - & 4687,5 & 5625,0 & 5156,3 & 5468,8 \\
\hline \multicolumn{6}{|c|}{ Análise de Variância (resumo) } \\
\hline \multirow{2}{*}{\multicolumn{2}{|c|}{$\begin{array}{l}\text { F (tratamento secundário dentro do cultivar) } \\
\text { C.V. }(\%)\end{array}$}} & $0,40^{\mathrm{ns}}$ & $0,76^{\mathrm{ns}}$ & $0,87^{\mathrm{ns}}$ & $0,25^{\text {ns }}$ \\
\hline & & \multicolumn{4}{|c|}{15,4} \\
\hline
\end{tabular}

i.a.: ingrediente ativo 
Tabela 5. Produção dos híbridos de milho tratados com diferentes herbicidas aplicados em pós emergência. Engenheiro Coelho, SP. $1997 / 1998$.

\begin{tabular}{|c|c|c|c|c|c|}
\hline \multirow{3}{*}{ Tratamento } & \multirow{3}{*}{$\begin{array}{c}\text { Dose } \\
\text { (g/ha) }\end{array}$} & \multicolumn{4}{|c|}{ Produção (kg/ha) } \\
\hline & & \multicolumn{4}{|c|}{ Híbridos } \\
\hline & & C-444 & C-505 & C-901 & C-909 \\
\hline Propisochlor & 1008 & 3437,5 & 5000,0 & 5312,5 & 6093,8 \\
\hline Propisochlor & 1080 & 4218,8 & 4062,5 & 5625,0 & 4531,3 \\
\hline Propisochlor & 1152 & 3750,0 & 6093,8 & 4062,5 & 5625,0 \\
\hline Propisochlor/atrazine & $1008 / 1400$ & 3750,0 & 5625,0 & 4062,5 & 4531,3 \\
\hline Propisochlor/atrazine & $1080 / 1400$ & 4062,5 & 5156,3 & 5625,0 & 5312,5 \\
\hline Propisochlor/atrazine & $1152 / 1400$ & 4062,5 & 5781,3 & 5468,8 & 5312,5 \\
\hline Propisochlor + terbutylazine & $1008+1800$ & 4062,5 & 5625,0 & 4531,3 & 5625,0 \\
\hline Propisochlor + terbutylazine & $1080+1800$ & 4062,5 & 6562,5 & 5937,5 & 6093,8 \\
\hline Propisochlor + terbutylazine & $1152+1800$ & 4218,8 & 5312,5 & 5312,5 & 6250,0 \\
\hline Acetochlor & 2688 & 3906,3 & 5000,0 & 4531,3 & 5625,0 \\
\hline Metolachlor & 1250 & 3593,8 & 5000,0 & 4218,8 & 5000,0 \\
\hline A trazine & 3500 & 4062,5 & 7031,3 & 4062,5 & 6250,0 \\
\hline Terbutylazine & 3000 & 3281,3 & 5000,0 & 3437,5 & 5937,5 \\
\hline Testemunha capinada & ---- & 4062,5 & 5312,5 & 5625,0 & 6250,0 \\
\hline \multirow{3}{*}{\multicolumn{2}{|c|}{$\begin{array}{l}\text { Análise de Variância (resumo) } \\
\text { F (tratamento secundário dentro do cultivar) } \\
\text { C.V }(\%)\end{array}$}} & & & & \\
\hline & & $0,14^{\mathrm{ns}}$ & 1,56 & $1,01^{\mathrm{ns}}$ & $0,81^{\mathrm{ns}}$ \\
\hline & & \multicolumn{4}{|c|}{30,8} \\
\hline
\end{tabular}

i.a.: ingrediente ativo.

\section{CONCLUSÕES}

(a) O herbicida propisochlor mostrou-se eficiente no controle das espécies Brachiaria plantaginea, Digitaria horizontalis, Eleusine indica, Amaranthus viridis e Bidens pilosa na dose mínima de $1008 \mathrm{~g} / \mathrm{ha}$, até 45 dias após sua aplicação em pré-emergência. (b) A adição de atrazine ou de terbutylazine ao propisochlor e aplicado em pré-emergência, incrementou consideravelmente o controle de $B$. plantaginea e $B$. decumbens, não interferindo no controle das demais espécies. (c) O herbicida propisochlor não deve ser recomendado para aplicação em pós emergência para $B$. plantaginea e $B$. decumbens, uma vez que nessa modalidade de uso, o controle dessas espécies foi insatisfatório. (d) Os híbridos de milho C444, C-505, C-901 e C-909, mostraram-se seletivos ao herbicida propisochlor não afetando suas produções nem ocasionando sintomas de injúrias por ação fitotóxica do produto, tanto quando aplicado em pré como em pós emergência.

\section{AGRADECIMENTOS}

Ao Eng ${ }^{\circ}$ Agrônomo M.A. Scarelis, da empresa Sipcam-Agro, pelas informações sobre o produto e demais facilidades para condução desse trabalho.

\section{LITERATURA CITADA}

ALMEIDA, J.C.V.; FORNAROLLI, D.A.; CHEHATA, A.N.; BRAZ, B.A.; BARROS, L.; COSTA, F.A. Eficiência e seletividade de herbicidas aplicados em pós-emergência inicial das plantas daninhas e da cultura de milho (Zea mays). In: CONGRESSO BRASILEIRO DEHERBICIDAS E PLANTAS DANINHAS, 17. Piracicaba, 1988. Resumos... Piracicaba, SP: SBCPD, 1988. p.255.

ASOCIACION LATINOAMERICANA DE MALEZAS. Recomendaciones sobre unificación de los sistemas de evaluación en ensayos de control de malezas. ALAM, Bogotá, v.1, n.1, p.35-38, 1974.

BLANCO, H.G.; ARAUJO, J.B.M.; OLIVEIRA, D.A. Estudo sobre a competição das plantas daninhas na cultura do milho (Zea mays, L.), determinação do período de competição. Arquivos do Instituto Biológico, São Paulo, v.43, n.3/4, p.105-114, 1976.

CARVALHO, J.A.; SANTOS, V.L.M.; SANTOS, C.M.; BRITO, C.H. Eficácia e seletividade dos herbicidas S-Metolachlor e S-Metolachlor mais atrazine na cultura do milho. In: CONGRESSO BRASILEIRO DA CIÊNCIA DAS PLANTAS DANINHAS, 22. Foz do Iguaçu, 2000. Resumos... Londrina: SBCPD, 2000. p.262.

CRUZ, J.C.; RAMALHO, M.A.P. Tração animal no controle de plantas daninhas na cultura do milho. In: EMPRESA BRASILEIRA DE PESQUISA AGROPECUÁRIACNPMS, Sete Lagoas, MG. Mecanização na cultura do milho, utilização da tração animal. Sete Lagoas, p.25-42, 1983. ( Circular Técnica, 09). 
Eficácia e seletividade do herbicida propisochlor aplicado em pré e pós-emergência, isolado e em misturas, sobre diferentes híbridos de milho

FOSTER, R. Farm Chemicals Handbook. Ohio: Meister Publishing, 1997. 850 p.

FRANS, R.; TALBERT, R.; MARX, D.; CROWLEY, H. Experimental design and techniques for measuring and analyzing plant responses to weed control practices. In: SOUTHERN WEED SCIENCE SOCIETY. Research Methods in Weed Science. 3 ed. 1986. p.29-46.
QUEIROZ, P.R.M. de; BARROS, A.C. de; MOURA, E. Efeito de herbicidas pré-emergentes no controle de plantas daninhas na cultura do milho (Zea mays L.), na região de Rio Verde/GO. In: CONGRESSO BRASILEIRO DA CIÊNCIA DAS PLANTAS DANINHAS, 21. Caxambu, 1997. Resumos... Viçosa, MG: SBCPD, 1997.p.223.

SILVA, J.B. da; SILVA, A.F. da. Controle bem para colher melhor. Sinal Verde, São Paulo, SP, v.2, n.4, p.12-13, 1987. 